\title{
Research of crack initiation in tension test specimen made of nickel alloy El961sh
}

\author{
Kamaliddin Karimbaev ${ }^{1, *}$, Ivan Pleshcheev ${ }^{1}$, and Elena Bredihina ${ }^{1}$ \\ ${ }^{1}$ Central Institute of Aviation Motors, Strength and dynamics Department, 111116, Russia
}

\begin{abstract}
In this paper method of numerical computations using explicit scheme, implemented in LSDyna (Ansys) software complex, is introduced and verified. Obtained solution explains experimental fact mentioned by P. Ludwik, that cracking in cylindrical test specimen made of plastic material starts in the middle of the smallest cross-section of the specimen. Introduced method allows verifying law, obtained by N.N. Davidenkov during unique experimental research in which he studied logarithmic strain in specimen's neck by pickling of cross-sections in this zone. Additionally it is possible to estimate amount of heat, generated during rupture of specimen, using obtained solution and law of conservation of energy. For this purpose tensile tests, in which thermal camera was used for temperature measuring, were conducted. It was shown, that all deformation energy apart from elastic and shape-forming energy in volume element, calculated in the moment before rupture is transformed to heat. Also tensile tests with various rate of loading were conducted for more detailed research of rupture process.
\end{abstract}

\section{Introduction}

Most strength properties of materials are obtained from standard tension test. Ductile materials usually crack after initiation of neck, when severe plastic deformation occurs. Strength limit is chosen as the largest stress, occurred during experiments. However, in various constructions (for example in disks) cracking occurs without necking. Therefore,other methods of calculating ultimate strengthare proposed. For example, in [1] true stress is proposed in form:

$$
S_{k}=\sigma_{f r} /(1-\psi)
$$

where, $S_{k}=P / A$ - ultimate fracture stress, $\sigma_{f r}=P / A_{0}-$ engineering fracture stress, $\psi$ - plasticity parameter, $P$ axial load, $A, A_{0}$ - actual and initial cross-section area respectively.

$P$. Ludwik [2] and other researchers showed by means of X-ray equipment, that cracking in cylindrical test specimen made of plastic material starts in the middle of the smallest cross-section of the specimen. After necking the stress state at the shrinking cross-section of the neck is not uniform and uniaxial anymore. That is why true stress obtained by (1) is not really true ultimate stress limit, but it is average axial stress. Experimental and numerical research of stress distribution in neck were introduced in this paper. Maximum axial stress in the middle of the smallest cross-section before fracture was computed. This value is proposed to be true ultimate stress limit.
In the secondsection method of numerical computations using explicit scheme, implemented in LSDyna (Ansys) software complex, is introduced and verified. Explicit scheme allows to adequately simulate severe plastic deformation, because it uses equation of motion instead of equilibrium equations. Due to the fact that in explicit scheme small integration time step is needed for achieving numerical stability, implicit scheme was used for calculations in elastic zone, while explicit scheme was used only after plastic yielding appeared. Computed stress distribution was compared with distribution, obtained by N.N. Davidenkov and Spiridonova [3] during experimental research in which they studied logarithmic strain in specimen's neck by pickling of cross-sections in this zone.

In the third section scale factor using specimens with different diameters of cross-section was investigated.

In the fourthsection formula based on the law of conversation of energy, for estimating amount of heat generated during rapture was proposed. To verify this formula tensile tests, in which thermal camera was used for temperature measuring, were conducted.

In the fifthsection dependence between true stress and loading rate was investigated

In the sixth section Poisson's ratio in deep plasticity was investigated using optical deformation measurements method (Vic-3D).

In the seventh section critique of modern methods of fracture mechanics is introduced. Usually crack is introduced in finite-element model after elastic solution is conducted, which might be inaccurate, because in plasticity stress distribution may change. 


\section{Method of identification of stress state in the smallest cross-section after necking.}

Method of calculating true ultimate stress proposed in this paper is based on the calculation of stress state using finite-element analysis of tension test of specimen. Finite element model of cylindrical test specimen is introduced in the figure 1 .

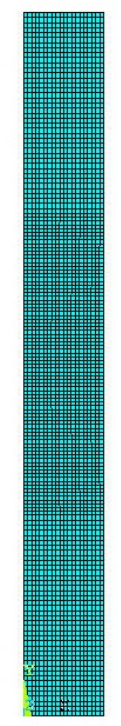

Fig. 1.Axisymmetric finite element model of specimen

Axisymmetric formulation of finite elements is used in this model. To archive necking small narrowing is introduced in the model (about 1\% reduction of radius of cylinder).As was mentioned before cracking of specimen occurs during severe plastic deformation, that is why it is necessary to use equation of motion. To use reduce computational time solution was conducted in two stages: implicit scheme was used for elastic deformation and explicit scheme for plastic yielding.In the explicit analysis in LS-Dyna static solution is used as preloading. Special numerical computation was conducted to assure that both explicit and implicit schemes provide the same results for elastic deformation.

Main difference of explicit analysis from implicit is that in explicit schemeuncoupled equation of motion is used and step by step solution is conducted. Equations used in Ls-Dyna explicit scheme are:

$$
\begin{aligned}
& \mathbf{U}_{t+\Delta t}=\mathbf{U}_{t}+\mathbf{V}_{t+\Delta t / 2} \Delta t_{t+\Delta t / 2} \\
& \mathbf{V}_{t+\Delta t / 2}=\mathbf{V}_{t-\Delta t / 2}+\mathbf{a}_{t} \Delta t_{t} \\
& \mathbf{a}_{t}=[\mathbf{M}]^{-1}\left(\mathbf{F}_{t}^{e x t}-\mathbf{F}_{t}^{i n t}\right) \\
& \mathbf{x}_{t+\Delta t}=\mathbf{x}_{0}+\mathbf{U}_{t+\Delta t} \\
& \Delta t_{c r}=2 / p_{\max }
\end{aligned}
$$

Where $\mathbf{U}_{t}$, - displacement vector for time $t$, $\mathbf{V}_{t+\Delta t / 2}$ - velocity vector for time $t+\Delta t / 2, \mathbf{a}_{t}-$ acceleration vector. $[\mathbf{M}]^{-1}-$ inverse mass matrix, $\mathbf{F}_{t}^{\text {ext }}$
,F $\mathbf{F}_{t}^{\text {int }}$ - external and internal force vector, $\Delta t_{c r}$ - critical time step, $p_{\max }-$ maximum frequency of model.

The base specimen is cylindrical specimen, made of EI961sh alloy with radius $\mathrm{r}=4 \mathrm{~mm}$. Stress-strain diagram obtained from experiment is shown in figure 2. Loading rate is $0.65 \mathrm{~mm} / \mathrm{s}$

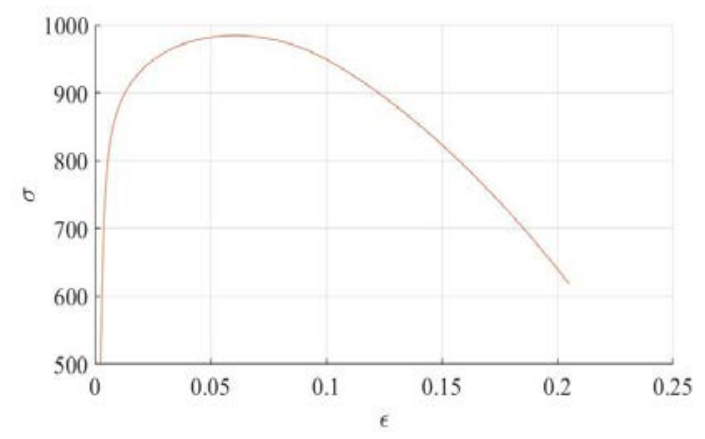

Fig.2.Experimental stress-strain diagram.

Finite element model (see figure 1) contains 3200 elements and 3381 nodes. Standard piecewise material model was used. True stress-strain diagram was obtained from experimental stress-strain diagram

However fracture stress and strain were adjusted so that the average axial stress before fracture and reduction at fracture were equal to those obtained from experiment. Average axial stress before fracture is calculated according (1) and is equal to $1630 \mathrm{MPa}$

Distribution of axial stress across the smallest cross-section before fracture is showed on the figure3. Ratio of maximum axial stress to average axial stress is 1.23 . It is suggested that maximum axial stress in the smallest cross-section is the actual true cracking stress and independent from size and construction of specimen.

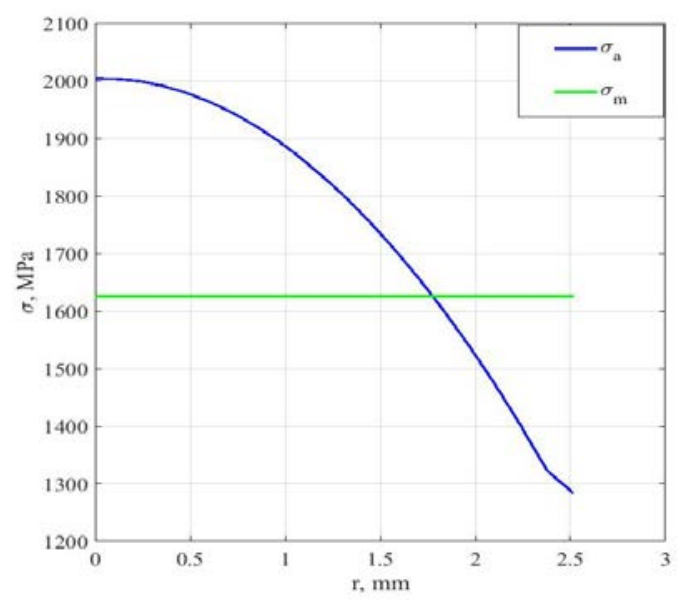

Fig.3.Axial stress distribution and average stress in the smallest cross-section

Davidenkov and Spiridonova, derived following formulas for stress distribution in the smallest crosssection:

$$
\begin{aligned}
& \sigma_{a}=\sigma_{i}(a / 2 R) \cdot\left(1-r^{2} / a^{2}+2 R / a\right) \\
& \sigma_{r}=\sigma_{t}=\sigma_{i}(a / 2 R) \cdot\left(1-r^{2} / a^{2}\right)
\end{aligned}
$$


Stress distribution from finite-element analysis was compared with distribution, derived by Davidenkov (see figure 4,5 ). Neck curative radius $R$ for Davidenkov's formula of distribution was calculated from FE model. Davidenkov's stress distribution is very closeto stress distribution derived by finite element analysis at the time $t=4.94$ seconds which is $\Delta t=3.06$ seconds before fracture. However for later times Davidenkov's stress distribution is much different from stress distribution obtained from finite element analysis.

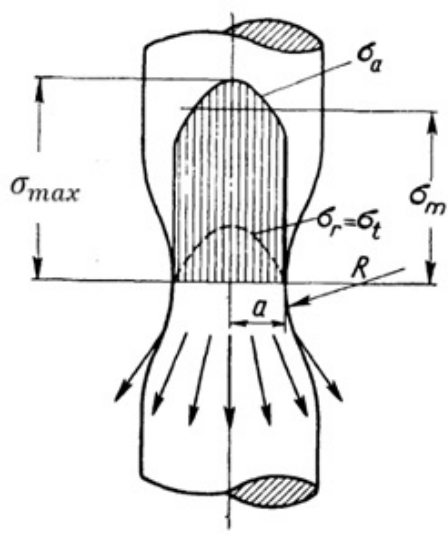

Fig.4.Stress distribution according Davidenkov's formula.

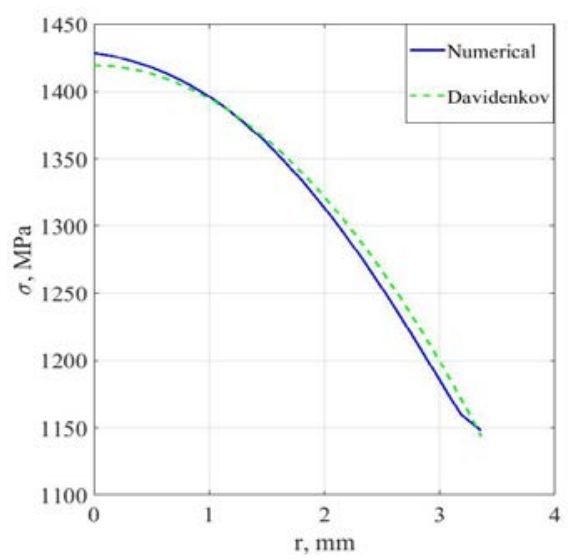

Fig.5.Axial stress distribution according numerical analysis and Davidenkov in the smallest cross-section

Since plastic deformation is dynamic process, stress is a function of time. That is why ratio of maximum axial stress in the specimen to average axial stress is not constant value and is changing during deformation.

\section{Scale factor}

It is widely known that in various materials true stress, calculated from (1) is decreasing as specimen radius increasing. Usually this fact is explained by statistics, the larger specimen, larger probability of having defects. However in this article it is shown that even in ideal material decreasing of medium axial stress with increasing radius will occur. It could be explained bythe fact that fracture occurs when the true stress limit is reached in the center of cross section. While true stress limit calculated by (1) is averaged axial stress, which means that it will be decreased when the area by which it is evaluated increases.

Figure shows axial stress distribution for different radiuses of specimen, obtained by numerical solution.

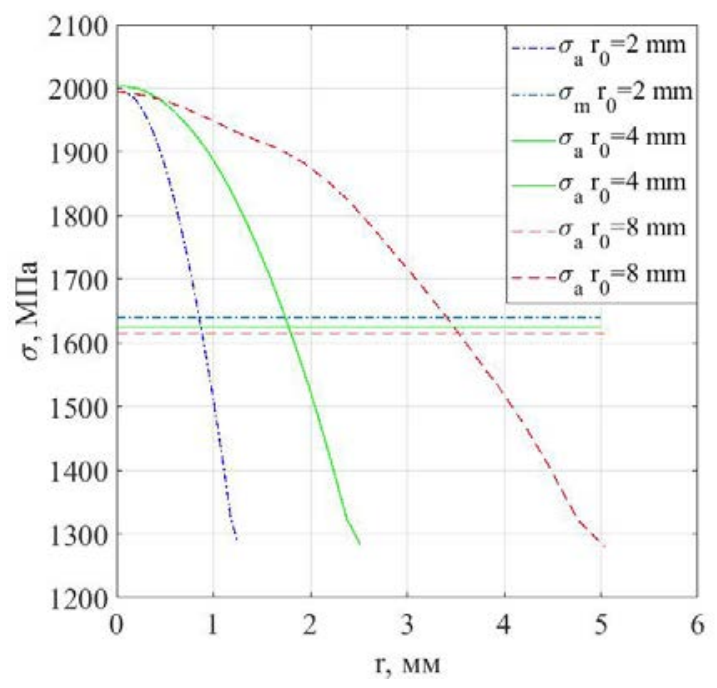

Fig.6.Axial stress distribution before rupture for various radiuses of specimen.

\section{Estimation of heat generated during rupture of specimen}

To estimate heat generation per volume unit during rupture following expression is proposed:

$$
Q=\sigma_{p r}\left(\varepsilon_{f r}-\varepsilon_{p r}\right)
$$

where $Q$ - heat generation per volume unit, $\sigma_{p r}-$ proportionality limit, $\varepsilon_{f r}$ - rupture deformation, $\varepsilon_{p r}-$ elastic deformation

This expression could be obtained by subtracting elastic energy and plastic distortion energy from full deformation energy:

$$
\begin{aligned}
& U_{\Sigma}=\int \sigma d \varepsilon \\
& U_{e l}=\sigma_{p r} \varepsilon_{p r} \\
& U_{d i s}=\int\left(\sigma-\sigma_{p r}\right) d \varepsilon
\end{aligned}
$$

where $U_{\Sigma}, U_{e l}, U_{d i s}-$ full, elastic and distortion deformation energy respectively.

Graphical representation of the result is shown on figure 7 . 


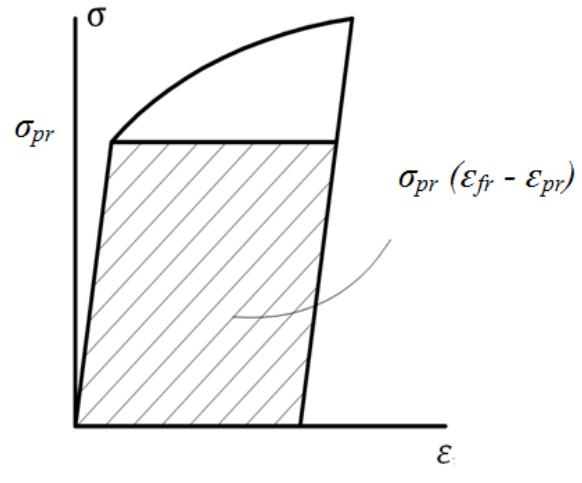

Fig.7.Illustration to formula (9)

To verify this formula tensile test, in which thermal camera was used for temperature measuring, was conducted.Frame frequency of thermal camera was equal to $p_{\text {cam }}=9 \mathrm{~Hz}$

Figure 8 shows temperatures measured by thermal camera during tests. Fracture occurred during time $0<\mathrm{t}_{\text {frac }}<1 / p_{\text {cam }}$. To estimate possible error of temperature measurements extrapolation was made (see Figure 8). Value of maximum measured temperature could be estimated within boundaries $54^{\circ} \mathrm{C}<\mathrm{T}_{\max }<59^{\circ} \mathrm{C}$. Value of temperature calculated by formula (9) is $\mathrm{T}_{\mathrm{cal}}=55 \cdot 4^{\circ} \mathrm{C}$.

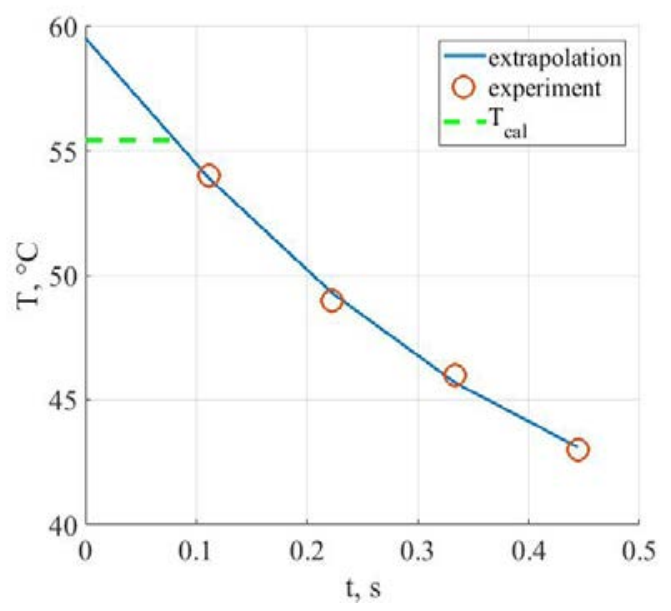

Fig.8.Temperature extrapolation.

\section{Effect of loading rate on true ultimate stress limit}

Loading rate could effect on true ultimate stress limit. Figure 9 shows 2 stress-strain curve obtained from experiment with various loading rates $(0.3 \mathrm{~mm} / \mathrm{min}$ and $0.65 \mathrm{~mm} / \mathrm{s}$ ). Radius of specimen's cross-section is $\mathrm{r}=4 \mathrm{~mm}$. Average axial stress before fracture was calculated (1) and equals $1620 \mathrm{MPa}$ and $1630 \mathrm{MPa}$ respectively.

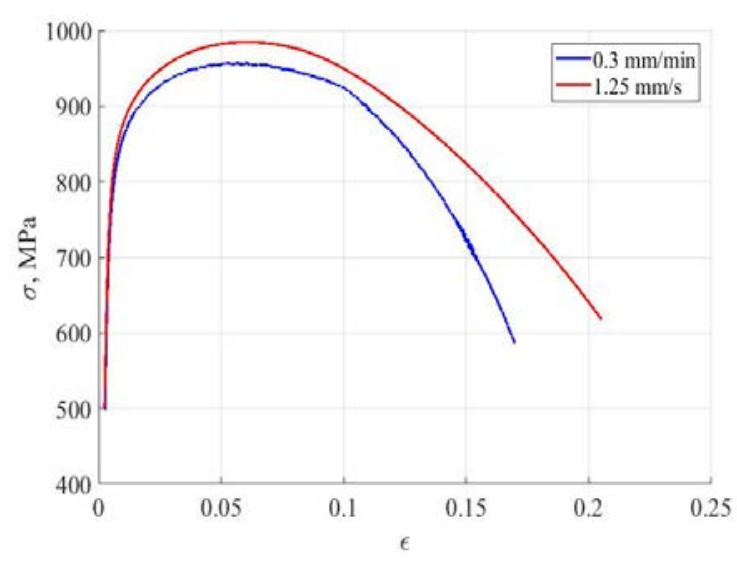

Fig.9.Experimental stress-strain diagram for various load rates.

Numerical experiments were conducted to investigate effect of loading rate upon true ultimate stress limit. Result is shown on the figure 10.

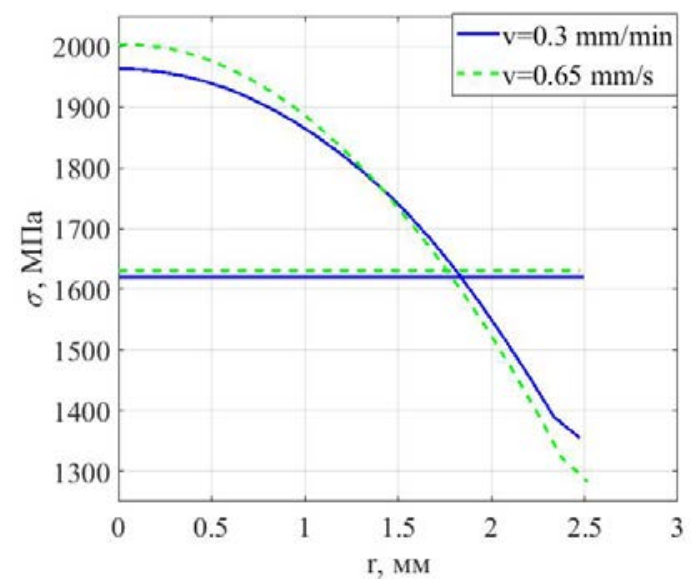

Fig.10.Axial stress distribution and average stress in the smallest cross-section for two different loading rates.

According to numerical simulations and experiments loading rate does not effect on true ultimate stress limit significantly.

\section{Poisson's ratio in deep plasticity}

Poisson's ratio in plasticity theory is usually suggested to be equal to $v=0,5$. In other words material in plastic state behaves like incompressible liquid. However this assumption is still to be verified.

To verify this assumption local axial and traverse strains should be measured. Standard tools of measurement like extensometers gauges are inapplicable in this case. Extensometers cannot measure local deformation. Strain gauges measure local deformation in a given point in the measuring surface area, however, it is very difficult to predict the point of yielding and the necking area. That is why optical measurement system was used (see figure 11). The system can measure local axial and traverse strains during tensile test (see figure 12). 


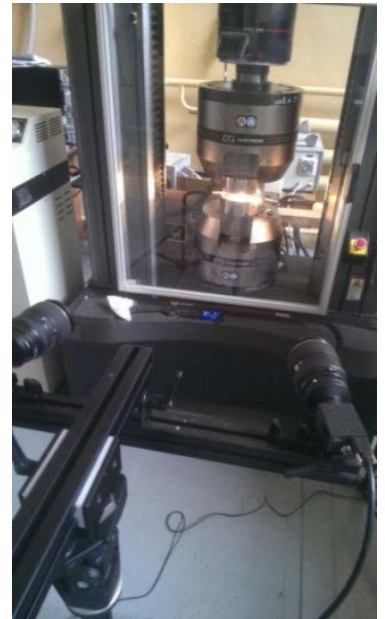

Fig.11.Vic-3D measurement system.

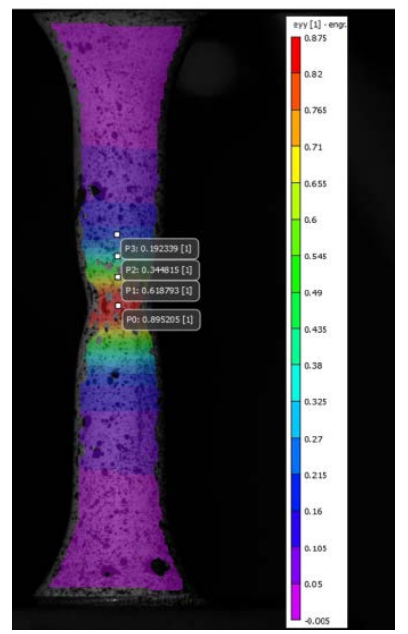

Fig.12.Picture of the specimen taken by optical system

Ratio of traverse strain to axial strain, obtained by optical measurement system for four surface points versus axial strain is shown in the figure 13 .

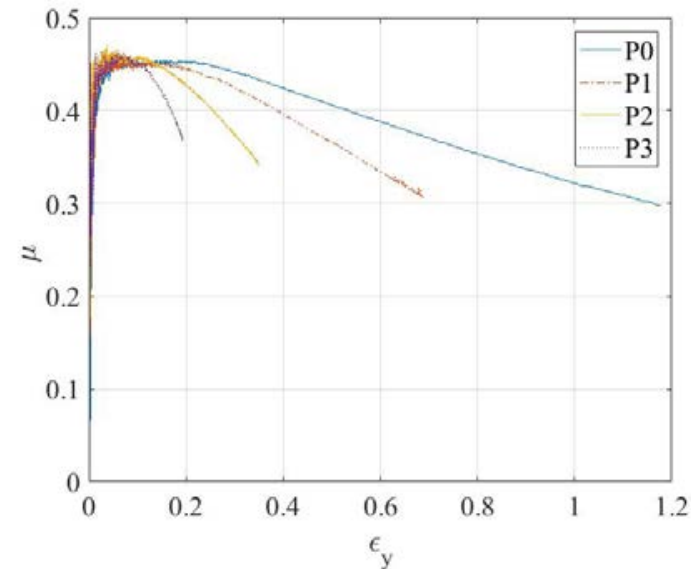

Fig.13.Poisson's ratio versus axial stress.

As can be seen Poisson's ratio changes during loading. In the end of loading Poisson's ratio is decreasing and is very much different than suggested $v=0.5$. That is why strain state calculated on this assumption might be inaccurate for EI916sh alloy.

\section{Conclusion (about using plasticity analysis in fracture mechanics)}

To evaluate life expectancy of construction with possible defects fracture mechanics is used.Modern fracture mechanics is based on elasticity theory to investigate stress state near the crack tip. In elastic approach crack tip becomes singularity point, in which infinite value of stress occurs.

To conduct solution correctly and consistently often computation of construction stress state without cracks is evaluated in elastic zone. After that cracks are introduced into zones, with largest stress values.It is known that stress distribution in elasticity theory is function, which is subject to the Cauchy conditions. Such function reaches an extremum at the boundaries of body. So after solving elastic problem for construction maximum stress occurs only at the boundariesof the body.

However, as one can see in the tension test specimen fracture starts in the center of specimen not at the boundaries. It means that if plasticity is reached during loading, stress redistribution occurs. Therefore stress distribution may be much different from distribution, obtained from elastic solution and maximum stress may occur in different location.

That is why for proper crack analysis plasticity should be taken into account. Though it is worthy of note that in real constructions presence of torque and bending load in elastic-plastic analysis leads to stress distribution with maximum stress at the body boundaries.

\section{Acknowledgments}

We wish to greatly acknowledge to Denis Palchikov for conducting experiment using optical measurement system and to Mikhail Volkov for thermal camera measurements of heat generation during rupture.

\section{References}

1. I.A. Birger, B.F. Shorr, (eds) (1993), Raschet na prochnost detaley mashin [Strength analysis of machine parts], Mashinostroenie, Moscow, Russia

2. P. Ludwik, Z. Ver. Deut. Ing., 71 (1927), 1532.

3. N.N. Davidenkov, N.I. Spiridonova, Mechanical methods of testing. Analysis of the state of stress in neck of a tension test specimen, American Society for Testing and Materials, 46, pp. 1147-1158, 1947 